\title{
LEVENTAMENTO DE PRÁTICAS DE DESENVOLVIMENTO DE PRODUTOS EM START-UPS DE HARDWARE EM UMA CIDADE DE SANTA CATARINA
}

Josue Basen Pereira (josuebasen@gmail.com) - Instituto Federal de Santa Catarina (IFSC)

Régis Kovacs Scalice (regis.scalice@ufsc.br) - Universidade Federal de Santa Catarina (UFSC)

\section{RESUMO}

As empresas denominadas startups enfrentam diversos desafios, em especial, aquelas que desenvolvem produtos físicos, as startups de hardware. Neste trabalho é objetivada análise do cenário atual do estado de Santa Catarina, particularmente a cidade de Joinville, em relação à maturidade das startups em relação ao conhecimento do Processo de Desenvolvimento de Produtos, visando avaliar se tal ambiente serve de laboratório a estudos voltados ao desenvolvimento de metodologias e ferramentas de projetos para startups. Foi realizado o levantamento de diferentes abordagens ao Processo de Desenvolvimento de Produto (PDP) considerando tanto os modelos clássicos quanto os voltados para startups. Uma pesquisa de campo foi empreendida onde um questionário foi aplicado a onze representantes de diferentes empresas deste tipo para se conhecer a realidade vivenciada por estas empresas no desenvolvimento de seus produtos. Os resultados obtidos indicam que a as startups analisadas na cidade de Joinville apresentam os mesmos problemas que os identificados na literatura em startups de hardware e, portanto, servem de laboratório para experimentação de iniciativas relacionadas ao desenvolvimento de métodos e ferramentas para o desenvolvimento de produtos voltados á esta categoria de empresas.

Palavras chave: desenvolvimento de produtos, startups de hardware, análise, questionário. 


\section{INTRODUÇÃO}

O número de empresas startups no Brasil mais do que dobrou no período entre 2011 e 2018 com a plataforma StartupBase, da Associação Brasileira de Startups (ABStartups), contabilizando em 2018 o cadastro de mais de seis mil startups no país (STARTUPBASE, 2018).

Não há consenso sobre a definição, mas o termo startup tem sido utilizado para designar empresas em fase inicial, com potencial de rápido crescimento, onde uma equipe busca desenvolver um produto ou serviço inovador, de base tecnológica, que proporcione um modelo de negócio replicável e escalável sem aumento proporcional dos seus custos (ABSTARTUPS, 2017). Tomando esta definição, para este trabalho uma startup será também considerada uma micro ou pequena Empresa de Base Tecnológica (EBT).

As micro e pequenas EBTs são micro e pequenas empresa que têm aptidão para desenvolver produtos, processos, modelos de negócio ou serviços inovadores (SEBRAE/IPT, 2001; MINISTÉRIO DA CIÊNCIA E TECNOLOGIA, 2018), têm uma alta proporção de gastos com pesquisa e desenvolvimento ( $\mathrm{P} \& \mathrm{D})$, empregam uma alta proporção de pessoal técnico e de engenharia e servem a mercados pequenos e específicos (JUGEND, 2006). Tomando como referência estas fontes, pode-se considerar EBT a empresa que têm como sua característica básica a aplicação de conteúdo tecnológico voltado para o sistemático desenvolvimento de novos produtos e atendem segmentos específicos (nichos) de mercado e/ou substituição de importações.

Dentre estas startups encontram-se as startups de hardware. De acordo com INOVAÇÃO SEBRAE MINAS (2017) toda startup que desenvolve produtos físicos passíveis de patente é uma startup de hardware. Para os fins deste trabalho startup de hardware e startup de produto físico serão considerados sinônimos. No contexto do estado Santa Catarina, uma pesquisa realizada com 329 startups verificou que apenas $36(11,61 \%)$ das empresas selecionaram a opção hardware, que está associada a produtos, para o tipo de serviço que prestam.

Em um cenário de mutações, pequenas e ágeis empresas com ideias inovadoras adquirem proeminência frente a grandes empresas com dificuldades de acompanhar as mudanças cada vez mais complexas e interconectadas do mercado e da sociedade (CALMADOVICI, 2011). As pequenas empresas que desenvolvem produtos inovadores têm se tornado importantes atores no mercado. Neste meio, as empresas de base tecnológica (EBTs), entre elas as startups de hardware estão presentes. Elas também empregam pessoal especializado, são geradoras ou 
potenciais geradoras de receita para o Estado, são importantes fatores na transferência dos conhecimentos da academia para a sociedade, além de agregar valor aos produtos e gerar riqueza tornando-se assim, importantes fatores da sociedade e da economia das nações onde se desenvolvem (SEBRAE/IPT, 2001).

Não obstante estas pequenas empresas tenham um papel relevante, elas padecem com uma série de dificuldades e desafios em suas atividades em geral e no desenvolvimento de produtos inovadores. Verificar quais são estas dificuldades e buscar soluções que potencializem os resultados do desenvolvimento de produtos inovadores por estas empresas se mostra como importante carência a ser sanada por estudos e pesquisas acadêmicas.

Para maximizar os resultados no desenvolvimento de produtos as empresas devem possuir um procedimento adequado que viabilize a aplicação de conhecimento técnico de um modo sistemático, buscando unir a ciência de projeto aos métodos científicos para analisar da melhor forma os sistemas técnicos e a sua interação com o ambiente e o usuário a fim de definir regras para o seu desenvolvimento (PAHL et al., 2005).

Pesquisa realizada por Costa Filho (2017) conclui que não há modelo de referência específico para o desenvolvimento de startups de hardware o que caracteriza uma dificuldade enfrentada por estas empresas. Ainda que esta conclusão possa ser confrontada, dadas as abordagens, como a de Einsten (2015), feitas nos últimos anos, permanece a necessidade de pesquisas que objetivem consolidar metodologias para estas empresas, em especial no contexto brasileiro. Considerando a importância crescente das startups e a lacuna das pesquisas quanto ao desenvolvimento de produtos em startups de hardware, observa-se a necessidade de entender o grau de conhecimento em práticas de desenvolvimento de produtos.

Neste trabalho é objetivada análise do cenário atual do estado de Santa Catarina, particularmente a cidade de Joinville, em relação à maturidade das startups em relação ao conhecimento do Processo de Desenvolvimento de Produtos, visando avaliar se tal ambiente serve de laboratório a estudos voltados ao desenvolvimento de metodologias e ferramentas de projetos para startups . A escolha da cidade é justificada pelo fato de Joinville ser a maior e o principal PIB industrial do estado.

\section{REVISÃO TEÓRICA}

O PDP consiste em um conjunto de atividades realizadas na busca por transformar uma ideia (identificada por meio de uma oportunidade ou necessidade) em um produto ou serviço que 
tenha valor no mercado. Este novo produto terá sucesso relativo ao sucesso da construção de um PDP eficiente e eficaz. A forma de se operacionalizar este processo se dá de forma sistematizada e segmentada em etapas que vão desde a ideia inicial até quando o produto é descontinuado.

Alguns autores (por exemplo PAHL et al., 2005; ROZENFELD et al., 2006, ULRICH \& EPPINGER, 2016) apresentam na literatura modelos de referência de PDP com a finalidade de orientar e proporcionar o controle neste processo. Cooper e Kleinschmidt (1995) e Griffin (1997) afirmam que a utilização de modelos de referência é uma das práticas mais usuais e necessárias para o sucesso dos produtos. Também afirmam que para a lucratividade dos novos produtos e para uma melhoria contínua do PDP de uma organização, o uso de um modelo de referência é um fator crítico.

Entretanto, considerando o contexto e as limitações existentes em pequenas empresas, há um reflexo direto no PDP, incluindo: a falta de planejamento de produto e portfólio, a ausência de um processo estruturado de desenvolvimento, a carência de uma cultura de gestão de projetos, os mecanismos de comunicação ineficientes e a dificuldade de cooperação entre as diferentes áreas da empresa (COOPER et al., 2001; LEDWITH, 2000). Some-se a isso, que as limitações de recursos humanos e financeiros muitas vezes acabam por prejudicar o perfil inovador característico destas empresas (NOKE e HUGHES, 2010).

Owens (2007), após observar as dificuldades do PDP de pequenas empresas, concluiu que os atrasos no desenvolvimento são devidos ao mau delineamento dos requisitos do produto, às incertezas relacionadas com a tecnologia, à falta de experiência dos gestores, como também aos recursos limitados.

Spomberg (2013) apresenta uma relação de quinze problemas detectados no desenvolvimento de produtos em pequenas empresas. Dentre esses problemas, destacam-se para este trabalho:

1. Não existe modelo de referência de PDP ou não é difundido

2. Falta um processo estruturado de tomada de decisão

3. Falta de planejamento do produto

4. O processo de desenvolvimento de produtos não é executado conforme planejado

5. Desconhecimento de melhores práticas de PDP

6. Ausência de conhecimentos de gestão de projetos

7. Falta ou ineficiência dos mecanismos de controle do processo como um todo 
As empresas de base tecnológica (EBTs) enfrentam dificuldades ligadas ao PDP, principalmente com a falta de uma cultura de uso de metodologia no desenvolvimento do produto (SCORALICK, 2005). Estudo realizado por Franco et al (2007) demonstra que estas empresas tem necessidade de treinamento em desenvolvimento do produto. Também se verificou que as EBTs estudadas tinham mais acesso a recursos voltados ao marketing de produtos e menos recursos voltados para a capacitação da equipe com vistas a desenvolver o produto.

Em se tratando de métodos focados em EBTs, algumas iniciativas foram encontradas na literatura (Jugend, 2006; Mendes, 2008; Kurumoto, 2009; Pinto, 2015). Entretanto, para o caso específico de startups de hardware foram encontrados apenas 3 modelos. Startups de hardware, de modo geral, não utilizam uma metodologia de desenvolvimento de produto, quer seja pela falta desta ou pelo desconhecimento (Einstein, 2015). A ausência de metodologia torna o processo caótico e altamente suscetível à ineficiência.

Dada sua importância a este projeto, será destacado o modelo para o projeto de produtos da Bolt, uma empresa de capital de risco focada em startups de hardware. Einstein (2015) apresenta, em linhas gerais, a metodologia para o PDP empregada pela Bolt, mostrado na Figura 1, o qual se divide quatro partes ou fases: Idealização (roxo), Design (laranja), Engenharia (azul) e Validação (verde). As fases de Design e Engenharia podem ocorrer de forma simultânea.

$\mathrm{Na}$ fase de ideação é realizada a pesquisa do problema, usualmente fruto de um vislumbre dos fundadores da startup. Após o levantamento de informações sobre o mercado a ser explorado, realizar conversas com possíveis clientes e gerar as personas que os representam, geram-se Protótipos de Prova de Conceito. São protótipos de baixo custo feitos de forma rápida para verificar a validação ou não de pressupostos e hipóteses que fundamentam a ideia do produto. Ao final desta fase a equipe deverá ter as hipóteses validadas antes de prosseguir para as fases seguintes. 


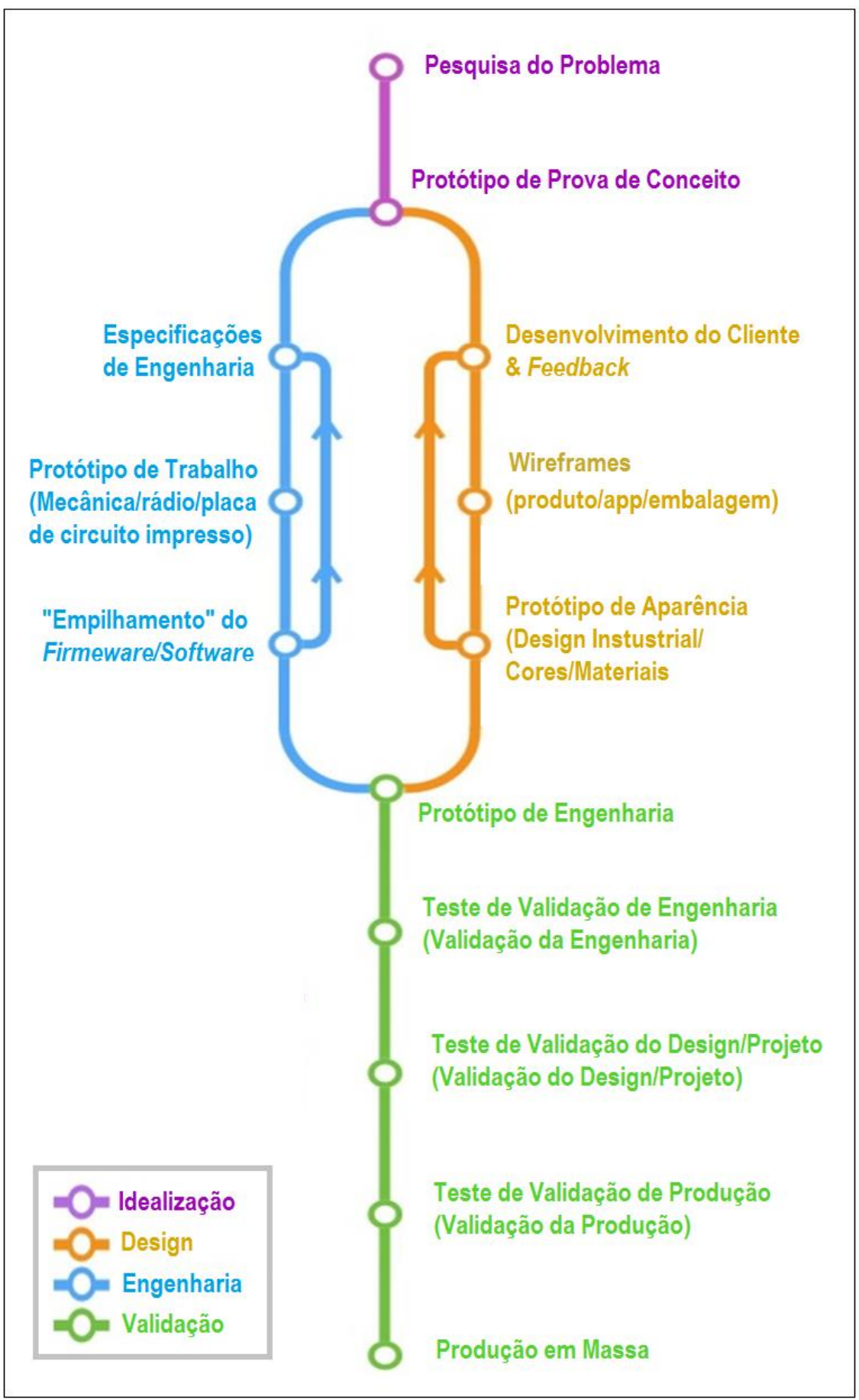

FIGURA 1 - Modelo Bolt de Desenvolvimento de Produto. Fonte: Einstein (2015).

A fase do Design se inicia com o Desenvolvimento do Cliente \& Feedback, é quando se realizam atividades com o objetivo de se compreender melhor a interação do cliente com o produto a fim de se obter informações que sirvam de base para o desenvolvimento do produto de forma mais ajustada as necessidades do cliente. Quando a equipe obtiver as informações necessárias, iniciará o desenvolvimento do Wireframe, um desenho esquemático básico que 
demonstra deforma clara a arquitetura de como o objeto (interface, página da internet, modelo, etc.) final será de acordo com as especificações relatadas. Também é gerado o storyboard para forçar a equipe a refletir sobre todo o ciclo de vida do produto incluindo embalagem, vendas, abertura da embalagem, configuração, primeiro uso, uso repetido ou casos de uso especial, suporte ao cliente, fim da vida. Por fim, a equipe precisará definir como será a aparência física do produto, e isso é realizado por meio da construção do Protótipo de aparência, incluindo formas, dimensões, cores, materiais, acabamentos, estabilidade e demais itens relativos a como o produto físico deverá se parecer.

A fase de Engenharia se inicia com a geração das Especificações de Engenharia, uma descrição técnica do produto para que este exerça a sua função. Na sequência passa-se a construção do Protótipo de Trabalho, um construto que realiza a função do produto atendendo as especificações de engenharia, já abrangendo componentes, placa de circuito impresso, modos de fabricação e preparação para o software embarcado. Finalizando a fase da Engenharia, realiza-se o "Empilhamento" do Firmware e do software, ou seja, realiza-se a programação do hardware do sistema embarcado. O resultado final é um protótipo realiza a função para a qual foi desenvolvido.

A fase de Validação inicia com a construção do Protótipo de Engenharia, que reúne o resultado das fases anteriores (aparência e funcionamento). Este protótipo é o maior passo no desenvolvimento do produto e com ele pode-se angariar recursos com os investidores. Também, ajustes são feitos e adequações para a fabricação e montagem.

Com o protótipo de engenharia finalizado, inicia-se o Teste de Validação de Engenharia onde é verificada a adequação do protótipo a exigências de agências reguladoras e demais órgãos fiscalizadores. Já no Teste de Validação da Produção, o sistema de produção é validado, certificando que grandes quantidades do produto poderão ser fabricadas, montadas e remetidas aos clientes.

Além do modelo da Bolt, outros modelos são encontrados na literatura focados em startups de hardware. O modelo da Bevator (MACÍAS, 2017) para o desenvolvimento de produto em startups é formada a partir de ferramentas e habilidades de Design Thinking, experiência do usuário, desenvolvimento ágil e Lean Startup. Nessa abordagem, primeiramente, se busca entender a oportunidade, como é o contexto da oportunidade observada, e conhecer o cliente em foco. Na definição da ideia, é necessário que o empreendedor defina qual é a vantagem competitiva de seu produto, como ela irá resolver os problemas do cliente, se ele pode construir 
um Produto Minimamente Viável (PMV) e quais alternativas o cliente utiliza atualmente. Por fim, é preciso validar o modelo de negócio junto ao mercado.

Outro modelo, da Gujarat Technology University (GTU), também se baseia no Design Thinking (OPENFUEL, 2014). O destaque desta proposta é ser uma metodologia didática, com atividades e ferramentas bem definidas para se obter uma ideia de produto adequada as necessidades do cliente. Ela inicia criando empatia com o possível usuário do produto a ser desenvolvido, listando suas atividades e criando histórias sobre o usuário. Em seguida, iniciase a Ideação aprofundando o conhecimento sobre as atividades do usuário, encontrando variações destas atividades. Dentro destas atividades podem-se identificar problemas que o usuário enfrenta e selecionar dentre eles os que serão abordados pela solução a ser proposta. Tecnologias e conceitos podem ser então elencados, uma lista de proposta de soluções pode ser montada e as soluções podem ser conectadas as atividades do usuário.

\section{METODOLOGIA}

O desenvolvimento da pesquisa seguiu as seguintes etapas: levantamento bibliográfico; entrevistas de pessoas com experiência prática no desenvolvimento de produtos em startups de hardware; organização do conhecimento levantado na pesquisa.

Para o estudo da literatura foi realizado o levantamento bibliográfico sobre metodologias de PDP específicas para startups e EBTs. Para a fase de entrevistas, a Incubadora 1 foi selecionada por critérios de conveniência, incluindo estar situada na região geográfica de Joinville, acessível para a realização do estudo, ser a mais antiga da região e ser incubadora com o maior número de startups na região. Um questionário foi aplicado a onze integrantes de diferentes startups de hardware, para verificar os conhecimentos de ferramentas ligadas ao PDP por parte dos integrantes destas empresas. Este questionário (APÊNDICE A) foi elaborado com base na revisão bibliográfica apresentada. O questionário também foi aplicado a 3 gerentes de desenvolvimento de produto de uma grande empresa multinacional para fins de comparação com os empresários das startups de hardware.

$\mathrm{Na}$ fase de Organização do Conhecimento, o conteúdo obtido foi utilizado para comparação entro o registrado na literatura e o observado na prática do PDP nas startups de hardware. 


\section{RESULTADOS E DISCUSSÃO}

Com o objetivo de se obter conhecimento prático sobre o como se dá o PDP em startups de hardware, foi selecionada a Incubadora 1 (nome fictício). Ela foi criada em 1995, tendo sediado em 1999 o primeiro mestrado de uma das universidades de Joinville, se consolidando como incubadora em 2001 e realizando as primeiras graduações de empresas em 2005. Desde então vem se posicionando como uma incubadora de base tecnológica tanto de hardware quanto de software contando, no ano de 2017, com mais de 65 empresas graduadas, 21 empresas incubadas sendo que destas, 13 são residentes e 8 virtuais (não estão presentes fisicamente nas instalações da incubadora). É uma fundação de caráter técnico científico, com personalidade jurídica de direito privado, sem fins lucrativos, mantida e dirigida por quatro entidades, duas universidades privadas, uma universidade pública e um sindicato ligado às empresas de processamento de dados e informática de Joinville cujos representantes formam a diretoria da incubadora sendo que estes diretores são voluntários.

Antes da aplicação do questionário, foi realizada uma palestra apresentando a metodologia utilizada na Bolt, escolhida por ser uma metodologia voltada para o desenvolvimento de hardware no contexto de uma startup e por ter disponível um exemplo de aplicação. $\mathrm{Na}$ sequência os representantes das empresas incubadas foram submetidos ao questionário disponível no Apêndice A, sendo os resultados apresentados na Figura 2.

Observa-se, inicialmente, que existem desenvolvedores em startups de hardware que desconhecem o que seria um Processo de Desenvolvimento de Produto (PDP) tradicional (5 em 11) e que ele é pouco utilizado mesmo que parcialmente nestas empresas. Este dado, bem como a visão geral acerca do nível de conhecimento e uso de ferramentas tradicionais de apoio ao PDP, como o QFD e FMEA, empresas confirma as observações de Owens (2007) e Spomberg (2013).

Destaca-se o que é constatado acerca do uso da abordagem do Lean Startup. Mesmo tendo 9 dos 11 startups conhecendo a abordagem, apenas uma delas afirma aplicar de forma integral esta abordagem enquanto que 7 delas afirmam aplicar parcialmente. Este dado confirma o que foi apurado na literatura acerca a dificuldade de se aplicar o ciclo de construir-testar-aprender proposto pelo Lean Startup no contexto de startups de hardware. 


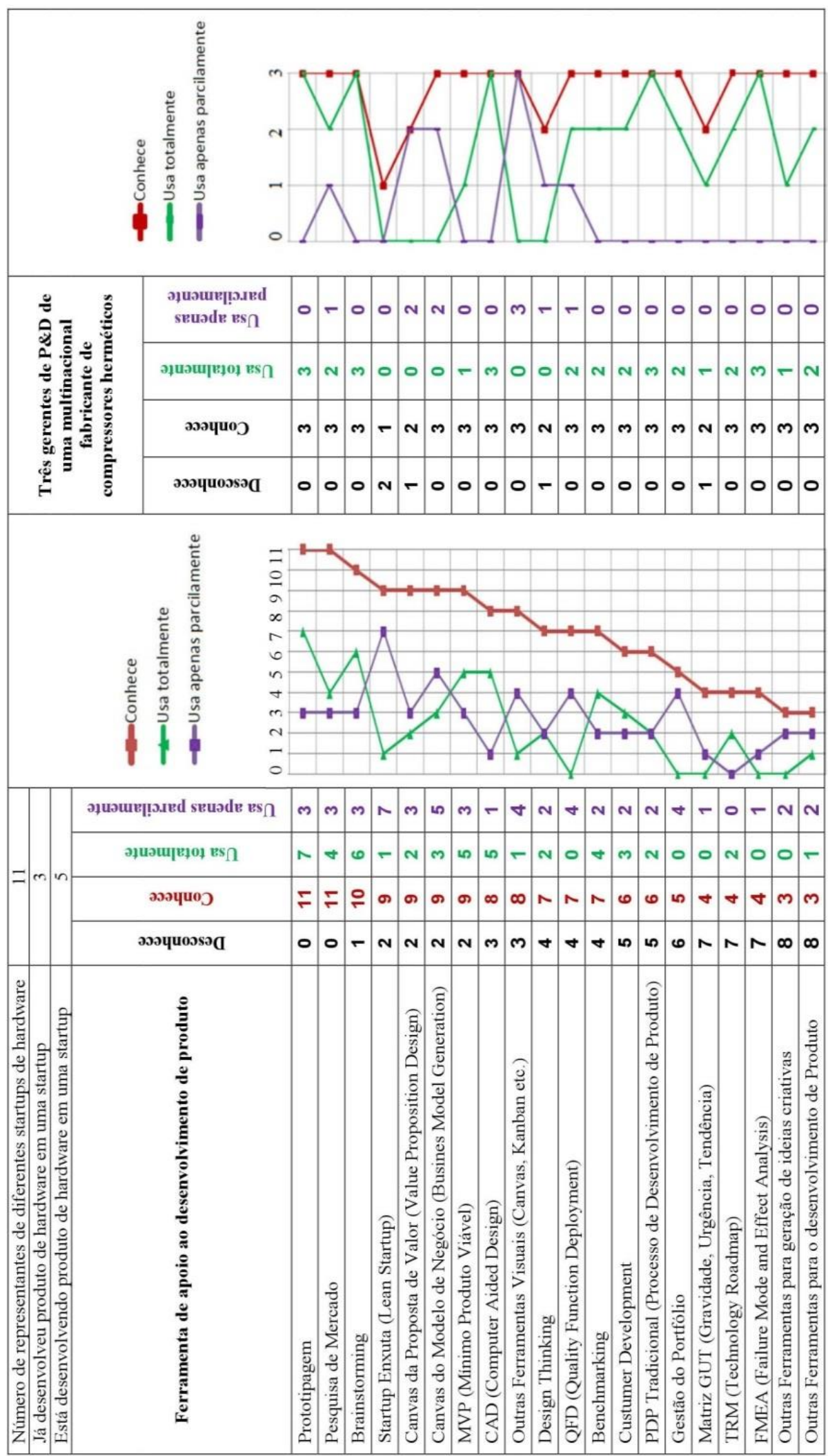

FIGURA 2 - Nível de conhecimento e aplicação de ferramentas de apoio ao PDP em 11 startups de hardware de Santa Catarina. Fonte: o autor 
A totalidade das empresas afirma conhecer e estar aplicando total ou parcialmente prototipagem. Por se tratar de empresas desenvolvendo produtos físicos, isso é natural, porém ao se comparar esta atividade prática com o nível de conhecimento e aplicação de ferramentas que dizem respeito ao posicionamento deste produto dentro de um negócio e na realidade do cliente e do mercado, pode-se argumentar que estas empresas estão mais preocupadas em construir um produto do que em construir uma proposta de valor robusta dentro de um modelo de negócio validado.

Salvo a diferença no tamanho das amostras, pode-se verificar uma substancial diferença no nível de conhecimento e aplicação das ferramentas entre as startups pesquisadas e os profissionais da grande empresa, evidenciando novamente o observado na literatura.

\section{CONCLUSÃO}

Este trabalho teve por objetivo avaliar se o cenário de startups de hardware na região de Joinville é equivalente ao observado na literatura. Para tanto, incialmente realizou-se um bibliográfico abrangendo as necessidades destas empresas, principalmente no tocante ao processo de desenvolvimento de produto. Por meio dele pôde-se verificar a existência de diferentes metodologias para o desenvolvimento de produto, tanto as genéricas para EBTs quanto as voltadas para startups, como também diferentes abordagens e ferramentas de apoio ao projeto e execução ao longo do desenvolvimento. Destaca-se a proposta de PDP para startups de hardware formulada por Pinto (2015) que buscou agregar em sua metodologia as contribuições ao desenvolvimento de produto que melhor viessem a servir no contexto das startups.

Em segundo momento foi verificado junto às empresas de uma incubadora de Joinville, como se dá o desenvolvimento de produtos em startups de hardware. Uma questionário foi aplicado à participantes de onze startups de hardware verificando-se o nível de conhecimentos dos mesmos sobre metodologias, abordagens e ferramentas para o desenvolvimento de produto. Estas pesquisas mostraram as necessidades dos envolvidos no processo. Observou-se que tal conhecimento é equivalente ao observado na literatura, onde se observa uma grande diferença entre o praticado por grandes empresas e o PDP das startups de hardware, bem como a presença dos mesmos problemas já registrados na literatura.

Desta forma, conclui-se que as startups de hardware da região de Joinville podem ser consideradas como laboratórios adequados para experimentação de novas abordagens 
metodológicas e ferramentas de projeto voltadas à solução de problemas observados nessa categoria de empresas.

\section{REFERÊNCIAS}

ABSTARTUPS. Tudo que você precisa saber sobre startups, 2017. Disponível em: https://abstartups.com.br/2017/07/05/o-que-e-uma-startup/. Acessado em 08/11/2018

CALMADOVICI, C. E. A inovação, a competividade e a projeção mundial das empresas brasileiras. Revista USP n ${ }^{\circ} 89$, p. 190-203, 2011.

COOPER, R. G. e EDGETT, S. J. e KLEINSHMIDT, E. Portfolio management for new product development: results of an industry practices study. R\&D Management, v. 31, n. 4, p. 361-380, 2001.

COOPER, R. G.; KLEINSCHMIDT, E. J. New Products Performance: Keys to Success, Profitability \& Cycle Time Reduction. Journal of Marketing Management, 11, p. 315-337, 1995.

COSTA FILHO, E. R. da; PEDROSO, M. C. Referências em modelos de desenvolvimento de startups de hardware. Anais. São Paulo: EAD/FEA/USP, 2017. Disponível em: http://sistema.emprad.org.br/2017/arquivos/124.pdf

EINSTEIN, Ben. The Illustrated Guide to Product Development. 2015. Disponível em: $<$ https://medium.com/@BenEinstein/the-illustrated-guide-to-product-development-part-1-ideationab797df1dac7>. Acesso em: 1 dez. 2017.

FRANCO, J. e colab. Principais dificuldades enfrentadas pelas empresas de base tecnológica: o caso das empresas da Incubadora Tecnológica de Maringá. 2007, Cuitiba: [s.n.], 2007. p. 14.

GRIFFIN, A. Modeling and measuring product development cycle time across intustries. Journal of product innovation management, v. 14, n. 6, p. 429-458, 1997.

INOVAÇÃO SEBRAE MINAS, Startups de Hardware: O Que São e Por Que Você Deve Ter Uma, 2017. Disponível em: http://inovacaosebraeminas.com.br/startups-de-hardware-o-que-sao-e-por-que-voce-deve-teruma/. Acessado em: 12/11/2018.

JUGEND, D. Desenvolvimento De Produtos em Pequenas e Médias Empresas de Base Tecnológica: Práticas de Gestão no Setor de Automação de Controle de Processos. 2006. 125 f. Universidade Federal de São Carlos, 2006.

KURUMOTO, J. S. Integração Entre Tecnologia e Produto nas Empresas de Base Tecnológica de São Carlos. 2009. 109 f. Universidade de São Paulo, 2009.

LEDWITH, A. Management of new product development in small eletronic firms. Journalof European Industrial Training, v. 24, p. 137-148, 2000.

MACÍAS, M. El camino para innovar: Cómo pasar de la idea al modelo de negocio creando valor para tus clientes (Sin colección). Bilbao: Deusto, 2017. 250 p.

MENDES, G. H. de S. O Processo de Desenvolvimento de Produto em Empresas de Base Tecnológica: Caracterização da Gestão e Proposta de Modelo de Referência. 2008. 294 f. Universidade Federal de São Carlos, 2008.

NOKE, H. e HUGHES, M. Climbing the value chain: strategies to create a new product capability in mature SMEs. International Journal of Operations \& Production Management, v. 30, n. 2, p. 132-154, 2010.

OPENFUEL, Help Manuel for 3rd semester for design thinking in engineering, 2014. Disponível em: https://pt.slideshare.net/kuntaldeshpande/learning-design-thinkingmanualfinal. Acessado em 12/11/2018.

OWENS, J. D. Why do some UK SMEs still find the implementation of a new product development process problematical? An exploratory investigation. Management Decision, v. 45, n. 2, p. 235-251, 2007.

PAHL, G. et al. Projeto na engenharia: fundamentos do desenvolvimento eficaz de produtos, métodos e aplicações. 6. ed. São Paulo: Edgard Blucher, 412 p. 2005. 
PINTO, R. S. Processo de desenvolvimento de novos produtos em startups: proposta de modelo. 2015. 171f. Dissertação (Mestrado em Engenharia de Produção) - Centro de Tecnologia, Universidade Federal do Rio Grande do Norte, Natal, 2015.

ROZENFELD, H, et al. Gestão de desenvolvimento de Produtos. 1. Ed. São Paulo: Saraiva, 2006.

SCORALICK, M. C. L.. Gestão do Processo de Desenvolvimento de Produto de Empresas de Pequeno Porte de Base Tecnológica de São Carlos: Estudo de Casos. 2005. 120 f. Dissertação de Mestrado em Eng. de Produção - Universidade Federal de São Carlos, São Carlos-SP, 2005.

SEBRAE/IPT. MPES de base tecnológica: conceição, formas de financiamento e análise de casos brasileiros. Relatório de Pesquisa, 2001.

SPOMBERG, T. K. Particularidades do Processo de Desenvolvimento de Produtos das Pequenas Empresas. 2013. 111 f. Universidade Federal do Rio Grande do Sul, 2013.

STARTUPBASE - Estatísticas, 2018. Disponível em: https://startupbase.abstartups.com.br/stats. Acessado em: $08 / 11 / 2018$

ULRICH, K. T. ; EPPINGER, S. D. Product Design and Development, $6^{a}$ edição, McGraw-Hill Companies, USA: 2016. 


\section{APÊNDICE A - Questionário de Pesquisa}

Nome:

E-mail:

Empresa: É uma startup de hardware? Sim ( ) Não ( )

Função:

Sobre desenvolver produto hardware em uma startup

Já desenvolveu（）Está desenvolvendo（）

1) Assinale abaixo o que é verdadeiro atualmente em sua empresa acerca das ferramentas da lista:

\begin{tabular}{|c|c|c|c|c|}
\hline$\frac{8}{0}$ & 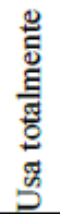 & 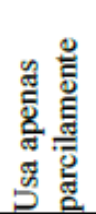 & Ferramenta de apoio ao desenvolvimento de produto & $\mathrm{N}^{\circ}$ \\
\hline & & & Startup Enxuta (Lean Startup) & 1 \\
\hline & & & Design Thinking & 2 \\
\hline & & & QFD (Quality Function Deployment) & 3 \\
\hline & & & Canvas da Proposta de Valor (Value Proposition Design) & 4 \\
\hline & & & Canvas do Modelo de Negócio (Busines Model Generation) & 5 \\
\hline & & & Matriz GUT (Gravidade, Urgência, Tendência) & 6 \\
\hline & & & MVP (Mínimo Produto Viável) & 7 \\
\hline & & & Prototipagem & 8 \\
\hline & & & Custumer Development & 9 \\
\hline & & & Pesquisa de Mercado & 10 \\
\hline & & & PDP Tradicional (Processo de Desenvolvimento de Produto) & 11 \\
\hline & & & TRM (Technology Roadmap) & 12 \\
\hline & & & CAD (Computer Aided Design) & 13 \\
\hline & & & Gestão do Portfólio & 14 \\
\hline & & & Benchmarking & 15 \\
\hline & & & Brainstorming & 16 \\
\hline & & & FMEA (Failure Mode and Effect Analysis) & 17 \\
\hline & & & Outras Ferramentas Visuais (Canvas, Kanban etc.). Quais? & 18 \\
\hline & & & Outras Ferramentas para geração de ideias criativas Quais? & 19 \\
\hline & & & Outras Ferramentas para o desenvolvimento de Produto . Quais? & 20 \\
\hline
\end{tabular}

